\title{
The Association between Leukocyte and Its Subtypes and Benign Breast Disease: The TCLSIH Cohort Study
}

\author{
Yanqi Song, ${ }^{1}$ Xuena Wang, ${ }^{2}$ Liyan Huang, ${ }^{2}$ Yeqing Gu, ${ }^{2}$ Xingqi Cao, ${ }^{2}$ Jingzhu Fu, \\ Hongmei $W u\left(\mathbb{D},{ }^{2}\right.$ Xiaojiang Li, ${ }^{1}$ Fanming Kong, ${ }^{1}$ Binxu Sun, ${ }^{1}$ Ruitian Sun, ${ }^{1}$ Qing Zhang, \\ Li Liu, ${ }^{3}$ Ge Meng, ${ }^{2,4}$ Shunming Zhang, ${ }^{2}$ Yingjie Jia $\mathbb{D}^{1},{ }^{1}$ and Kaijun Niu $\mathbb{D}^{2,3,5,6}$ \\ ${ }^{1}$ First Teaching Hospital of Tianjin University of Traditional Chinese Medicine, Tianjin, China \\ ${ }^{2}$ Nutritional Epidemiology Institute and School of Public Health, Tianjin Medical University, Tianjin, China \\ ${ }^{3}$ Health Management Centre, Tianjin Medical University General Hospital, Tianjin, China \\ ${ }^{4}$ Department of Toxicology and Sanitary Chemistry, School of Public Health, Tianjin Medical University, Tianjin, China \\ ${ }^{5}$ Tianjin Key Laboratory of Environment, Nutrition and Public Health, Tianjin, China \\ ${ }^{6}$ Center for International Collaborative Research on Environment, Nutrition and Public Health, Tianjin, China
}

Correspondence should be addressed to Yingjie Jia; jiayingjie1616@sina.com and Kaijun Niu; nkj0809@gmail.com

Received 18 February 2020; Accepted 13 May 2020; Published 31 May 2020

Academic Editor: Reza Izadpanah

Copyright (c) 2020 Yanqi Song et al. This is an open access article distributed under the Creative Commons Attribution License, which permits unrestricted use, distribution, and reproduction in any medium, provided the original work is properly cited.

\begin{abstract}
Inflammation plays a crucial role in the formation of benign breast disease. Given the limited study to explore the association between leukocyte as an indicator of immune system and benign breast disease, we used data from a large cross-sectional study to investigate association between leukocyte and its subtypes and benign breast disease among women in the general population. The data were derived from baseline data of the Tianjin chronic low-grade systemic inflammation and health (TCLSIH) cohort study during 2014 and 2016. Breast thickness and nodules status were assessed by using ultrasonography. Leukocyte and its subtype counts were carried out using the automated hematology analyzer. Multiple logistic regression analysis was used to examine the association between leukocyte and its subtypes and prevalence of benign breast disease. In the present study, the prevalence of benign breast disease was $20.9 \%$. After adjustments for potentially confounding factors, the odds ratios ( $95 \%$ confidence interval) for benign breast disease across lymphocyte quintiles were as follows: 1.00 (reference), $0.99(0.82,1.2), 0.85$ (0.69, 1.04$), 0.84$ (0.68, $1.02)$, and $0.75(0.61,0.92)(P$ for trend $=0.002)$. An inverse association between lymphocyte counts and benign breast disease was found, but leukocyte and other subtypes have nothing to do with benign breast disease. Further prospective studies are needed to determine the findings.
\end{abstract}

\section{Introduction}

Breast cancer $(\mathrm{BC})$ is a commonly diagnosed malignancy and the leading cause of cancer death in women worldwide [1]. Benign breast disease (BBD), the hallmark of which is epithelial proliferation, is a putative BC precursor [2]. BBD refers to a galaxy of pathophysiologic lesions resulting from progressive/regressive changes involving the component mammary structures, i.e., ducts, acini, stroma, and fat tissue [3]. The etiology of BBD is poorly characterized. Increasing evidence suggests that cancer-related inflammatory response plays a critical role in the development and progression of several malignancies. For instance, impairment of adaptive immune responses during chronic inflammation may favor tumor growth, angiogenesis, and cancer cell survival [4-8]. Previous studies have also indicated that inflammation pathways may be associated with $\mathrm{BBD}$, from which early stage BC may develop $[2,9]$.

The primary response cells to inflammation are leukocytes. Leukocytes are the main building blocks of the immune system. The immune defense against tumors is largely a cell-mediated one and relies on the integrity of $\mathrm{T}$ cell subsets [9]. Meanwhile, B cells can also mount humoral immune response with antitumor effects [9]. Furthermore, 
different leukocyte counts reflect different levels of inflammation [10]. In past studies, researchers have found that different leukocyte counts are strongly associated with different types of cancer, such as endometrial cancer, lung cancer, endometrial cancer, and BC [11-13]. However, to the best of our knowledge, no previous studies have investigated the association between leukocyte and its subtypes and BBD in adult women.

Therefore, we designed a cross-sectional study to investigate how leukocyte and its subtypes are associated with the prevalence of $\mathrm{BBD}$ among women in the general population.

\section{Materials and Methods}

2.1. Study Population. The study sample was taken from participants in the Tianjin chronic low-grade systemic inflammation and health (TCLSIH) cohort, details of which have been published elsewhere [14]. This study conformed to the ethical guidelines of the 1975 Declaration of Helsinki. The protocols and procedures of the study were approved by the Institutional Review Board of Tianjin Medical University with the reference number of TMUhMEC 201430, and written informed consent was obtained from each participant.

During the research period, 5,954 participants, who received health examinations including body mass index (BMI), waist, a series of blood parameters, and breast ultrasonography and had completed questionnaires regarding their smoking and drinking habits and disease history over the course of January 2014 to December 2016, were recruited. We excluded participants who did not undergo leukocyte counts $(n=23)$. Participants with a history of cancer $(n=89)$ were also excluded. After these exclusions, the final cohort study population comprised 5, 842 participants (Figure 1).

\subsection{Assessment of Leukocyte and Its Subtype Counts.} Fasting blood samples were gained from all the participants by venipuncture of the cubital vein in the morning and immediately mixed with EDTA. Leukocyte and its subtype counts were carried out using the automated hematology analyzer XE-2100 (Sysmex, Kobe, Japan) and expressed as $\times 1,000$ cells $/ \mathrm{mm}^{3}$. The test for blanks was $\leq 0.2 \times 10^{9}$ cells $/ \mathrm{L}$; the intra- and interassay coefficients of variation $(\mathrm{CV})$ were $\leq 2.0 \%$; and the cross-contamination rate was $\leq 0.5 \%$. In order to investigate how the leukocyte and its subtypes are associated with prevalence of BBD, we divided participants into quintiles based on leukocyte and its subtype counts.

2.3. Assessment of $B B D$. For the early screening and diagnosis of breast disease, both breasts were examined by breast ultrasound in the present study [15]. Breast ultrasonography was performed by trained sonographers using an iU22 ultrasonography system (Royal Philips) equipped with a $50 \mathrm{~mm}$ linear array transducer and a bandwidth of $7-12 \mathrm{MHz}$ to measure breast gland thickness and status of breast nodule (position, size, and nature) [16]. All participants were asked to stay in the supine position and

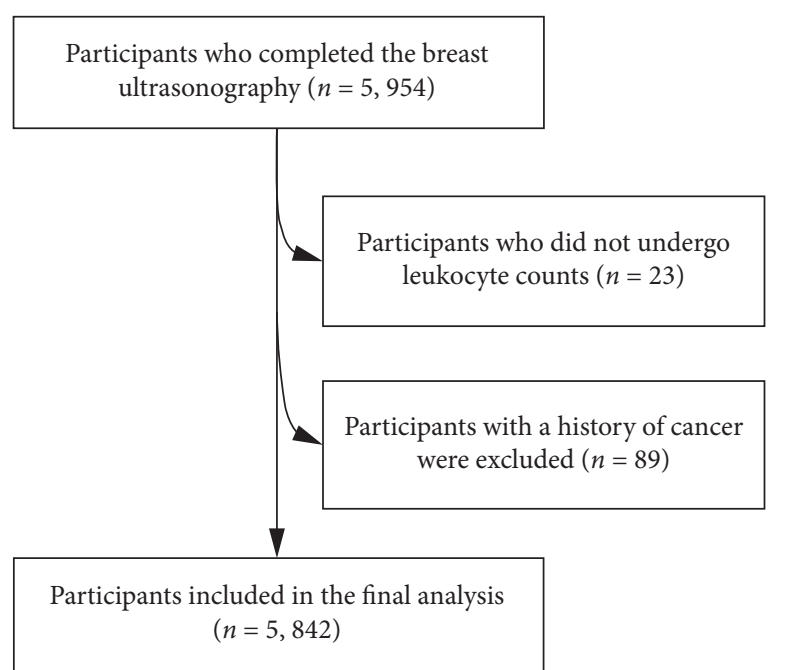

Figure 1: Flow diagram showing the selection of the study population.

exposed breast. Arms are naturally lifted that bilateral breast and axillaries were fully exposed. Bilateral breasts were measured in turn. Measured indicators include breast thickness and breast nodule size. The lesions were classified as simple cyst, complicated cyst, fibrocystic changes, sonographically benign solid lesion (mass), and ductal ectasia. If an ultrasonographic image owned the features of more than one of any of the abovementioned lesions, then it was named as a mixed lesion [17]. Imaging results were also categorized into five levels using the Breast Imaging Reporting and Data System (BI-RADS) diagnostic category [18]. The intra- and inter-measure coefficients of variability $(\mathrm{CV})$ were $<2.9 \%$.

2.4. General Examination. The anthropometric indicators (height, body weight, and waist circumference (WC)) were measured by well-trained investigators using a standard protocol. Waist circumference was measured at the umbilical level with subjects standing and breathing normally. The thresholds used to determine abdominal obesity were defined as $W C \geq 80 \mathrm{~cm}$ for Chinese women. BMI was calculated as weight $/$ height ${ }^{2}\left(\mathrm{~kg} / \mathrm{m}^{2}\right)$ [19]. Obesity was defined as $\mathrm{BMI} \geq 28 \mathrm{~kg} / \mathrm{m}^{2}$ according to the criteria of the Working Group on Obesity in China (WGOC) [19].

The sociodemographic variables including age, disease history, family history as well as current medication, menstrual and reproductive history (age at menarche, parity, age at first full-term birth, and menopausal status), smoking (defined as 'current smoker,' 'ex-smoker,' and 'nonsmoker'), and drinking status (defined as 'everyday,' 'sometimes,' 'exdrinker,' and 'nondrinker') were noted by self-reported form on a questionnaire. If women were ex-smokers or exdrinkers, they were asked age at which they had ceased smoking or drinking. Ex-smokers or ex-drinkers were defined as not smoking or drinking in a month prior to baseline, but used to be drinker and smoker. Occasional drinkers were defined as except for daily drinkers and drinking frequency more than one time in the past month 
TABle 1: Age-adjusted subjects characteristics by BBD status $(n=5,842)^{\mathrm{a}}$.

\begin{tabular}{|c|c|c|c|}
\hline \multirow{2}{*}{ Characteristics } & \multicolumn{2}{|c|}{ BBD } & \multirow{2}{*}{$P$ value } \\
\hline & No & Yes & \\
\hline No. of subjects & 4621 & 1221 & - \\
\hline Age $(y)$ & $47.9(47.8,48.0)^{\mathrm{c}}$ & $48.5(48.4,48.6)$ & $<.0001$ \\
\hline BMI $(\mathrm{kg} / \mathrm{m} 2)$ & $23.9(23.8,24.0)$ & $23.6(23.4,23.8)$ & $<.001$ \\
\hline $\mathrm{WC}(\mathrm{cm})$ & $80.3(80.0,80.5)$ & $79.0(78.6,79.5)$ & $<.0001$ \\
\hline $\mathrm{TC}(\mathrm{mmol} / \mathrm{L})$ & $4.95(4.92,4.97)$ & $4.95(4.90,5.00)$ & 0.88 \\
\hline TG $(\mathrm{mmol} / \mathrm{L})$ & $1.10(1.08,1.11)$ & $1.09(1.06,1.11)$ & 0.40 \\
\hline LDL-C (mmol/L) & $2.83(2.81,2.86)$ & $2.85(2.80,2.89)$ & 0.62 \\
\hline HDL-C $(\mathrm{mmol} / \mathrm{L})$ & $1.47(1.46,1.48)$ & $1.48(1.46,1.50)$ & 0.27 \\
\hline $\mathrm{SBP}(\mathrm{mmHg})$ & $121.0(120.6,121.5)$ & $119.4(118.6,120.3)$ & 0.001 \\
\hline DBP (mmHg) & $74.6(74.3,74.9)$ & $74.2(73.7,74.8)$ & 0.22 \\
\hline FBG $(\mathrm{mmol} / \mathrm{L})$ & $5.07(5.05,5.10)$ & $5.06(5.02,5.10)$ & 0.61 \\
\hline Smoking status (\%) & $12.3(11.4,13.2)$ & $11.3(9.54,13.3)$ & \\
\hline Smoker & 3.10 & 2.90 & 0.94 \\
\hline Ex-smoker & 0.71 & 0.35 & 0.25 \\
\hline Non-smoker & 96.2 & 96.7 & 0.68 \\
\hline \multicolumn{4}{|l|}{ Drinker (\%) } \\
\hline Everyday & 0.58 & 1.32 & 0.02 \\
\hline Sometimes & 34.5 & 37.2 & 0.23 \\
\hline Ex-drinker & 5.67 & 6.94 & 0.21 \\
\hline Non-drinker & 59.3 & 54.4 & 0.03 \\
\hline Menopausal status & 53.1 & 43.7 & 0.11 \\
\hline History of surgery (\%) & 2.01 & 2.78 & 0.03 \\
\hline History of CVD (\%) & 6.15 & 4.10 & 0.75 \\
\hline
\end{tabular}

BBD, benign breast disease; BMI, body mass index; WC, waist circumference; TC, total cholesterol; TG, triglycerides; LDL-C, low-density lipoprotein cholesterol; HDL-C, high-density lipoprotein cholesterol; SBP, systolic blood pressure; DBP, diastolic blood pressure; FBG, fasting blood glucose; CVD, cardiovascular disease., ${ }^{a}$ Analysis of covariance or multiple logistic regression analysis. ${ }^{b}$ Boldface indicates statistical significance $(P<0.05)($ or appropriate value). ${ }^{c}$ Geometric mean ( $95 \%$ confidence interval) (all such values).

prior to baseline. The history of surgery was assessed according to the responses to relevant questions and the personal health records.

All blood tests were performed after an overnight. As for lipids, total cholesterol (TC) and triglycerides (TG) were measured by enzymatic methods. Low-density lipoprotein (LDL) was measured by the polyvinyl sulfuric acid precipitation method and high-density lipoprotein (HDL) was measured by the chemical precipitation method. Levels of fasting blood glucose (FBG) were measured by glucose oxidase method. All these measurements were conducted by using appropriate kits on a Cobas 8000 automatic biochemistry analyzer (Roche, Mannheim, Germany). Blood pressure (BP) was measured twice in the right arm using an automatic device (TM-2655P, A\&D Company, Ltd., Tokyo, Japan) after 5 minutes of rest in a seated position. The mean of two measurements was taken as the BP value.

2.5. Statistical Analysis. All statistical analyses in the present study were performed using the Statistical Analysis System 9.3 edition for Windows (SAS Institute). In this study, all continuous variables were not normally distributed by using the Kolmogorov-Smirnov test. The log transformation for those nonnormal distribution variables was applied before the statistical analysis. The continuous variables are presented as the geometric means and 95\% confidence interval (CI) after logarithmic transforming, and categorical variables are shown as percentages. For analysis, the BBD statuses were used as dependent variables, and the quintiles of leukocyte and its subtype counts were used as independent variables. For characteristics analysis, differences among BBD statuses were examined using ANCOVA for continuous variables or using multiple logistic regression analysis for categorical variables after adjustment for age. Associations between different leukocyte and its subtypes counts and BBD were examined using logistic regression in three different models, and odds ratio (OR) and 95\% CI were calculated. Analysis was performed without any adjustment in model 1; the analysis was adjusted for age and BMI in model 2; model 3 additionally adjusted for smoking status, drinking status, history of surgery, menopausal status, hypertension, hyperlipidaemia, diabetes, and the metabolic syndrome as well as for family history of CVD (cerebrovascular disease). The median value of each leukocyte subtype quintile was used to calculate the $P$ values for linear trends. Interactions between different leukocyte subtypes counts, menopausal status, and confounders of BBD were tested by addition of cross-product terms to the regression model. The analysis applied a two-tailed significance test and considered $P<0.05$ as an indication of statistical significance.

\section{Results}

Among 5,842 individuals, 1,221 participants (20.9\%) met the criteria of $\mathrm{BBD}$. The characteristics of the study participants according to no $\mathrm{BBD}$ and $\mathrm{BBD}$ status are 
TABLE 2: Adjusted associations between categories of leukocyte and its subtypes and prevalence of benign breast disease ${ }^{\mathrm{a}}$.

\begin{tabular}{|c|c|c|c|c|c|c|}
\hline & \multicolumn{5}{|c|}{ Quintiles of leukocyte and its subtypes concentration $\left(\times 10^{9} / \mathrm{L}\right)$} & \multirow{2}{*}{$P$ for trend $\mathrm{d}^{\mathrm{b}, \mathrm{g}}$} \\
\hline & Level 1 & Level 2 & Level 3 & Level 4 & Level 5 & \\
\hline $\begin{array}{l}\text { Serum leukocyte concentration } \\
\left(\times 10^{9} / \mathrm{L}, \text { range }\right)\end{array}$ & $3.92-4.71$ & $4.71-5.40$ & $5.40-6.10$ & $6.10-7.40$ & $\geq 7.40$ & \\
\hline No. of subjects & 1170 & 1241 & 1048 & 1180 & 1203 & - \\
\hline No. of BBD & 258 & 272 & 207 & 242 & 242 & - \\
\hline Model $1^{c}$ & 1.00 (reference) d & $\begin{array}{c}0.99 \\
(0.82,1.20)\end{array}$ & $\begin{array}{c}0.87 \\
(0.71,1.07)\end{array}$ & $\begin{array}{c}0.91 \\
(0.75,1.11)\end{array}$ & $\begin{array}{c}0.89 \\
(0.73,1.08)\end{array}$ & 0.18 \\
\hline Model $2^{\mathrm{e}}$ & 1.00 (reference) & $\begin{array}{c}1.01 \\
(0.83,1.23)\end{array}$ & $\begin{array}{c}0.90 \\
(0.73,1.10)\end{array}$ & $\begin{array}{c}0.95 \\
(0.78,1.16)\end{array}$ & $\begin{array}{c}0.93 \\
(0.76,1.14)\end{array}$ & 0.40 \\
\hline Model $3^{\mathrm{f}}$ & 1.00 (reference) & $\begin{array}{c}1.01 \\
(0.83,1.22) \\
\end{array}$ & $\begin{array}{c}0.90 \\
(0.73,1.11) \\
\end{array}$ & $\begin{array}{c}0.94 \\
(0.77,1.16) \\
\end{array}$ & $\begin{array}{c}0.92 \\
(0.75,1.14) \\
\end{array}$ & 0.37 \\
\hline $\begin{array}{l}\text { Serum neutrophil concentration } \\
\left(\times 10^{9} / \mathrm{L}, \text { range }\right)\end{array}$ & $2.00-2.58$ & $2.58-3.05$ & $3.05-3.60$ & $3.60-4.63$ & $\geq 4.63$ & \\
\hline No. of subjects & 1193 & 1161 & 1101 & 1215 & 1172 & - \\
\hline No. of BBD & 266 & 232 & 218 & 245 & 260 & - \\
\hline Model $1^{\mathrm{c}}$ & 1.00 (reference) & $\begin{array}{c}0.87 \\
(0.71,1.06)\end{array}$ & $\begin{array}{c}0.86 \\
(0.70,1.05)\end{array}$ & $\begin{array}{c}0.88 \\
(0.72,1.07)\end{array}$ & $\begin{array}{c}0.99 \\
(0.81,1.21)\end{array}$ & 0.82 \\
\hline Model $2^{\mathrm{e}}$ & 1.00 (reference) & $\begin{array}{c}0.90 \\
(0.74,1.10)\end{array}$ & $\begin{array}{c}0.89 \\
(0.72,1.08)\end{array}$ & $\begin{array}{c}0.92 \\
(0.75,1.12)\end{array}$ & $\begin{array}{c}1.04 \\
(0.85,1.26)\end{array}$ & 0.53 \\
\hline Model $3^{\mathrm{f}}$ & 1.00 (reference) & $\begin{array}{c}0.89 \\
(0.73,1.09) \\
\end{array}$ & $\begin{array}{c}0.88 \\
(0.72,1.08) \\
\end{array}$ & $\begin{array}{c}0.91 \\
(0.74,1.11)\end{array}$ & $\begin{array}{c}1.01 \\
(0.82,1.24) \\
\end{array}$ & 0.71 \\
\hline $\begin{array}{l}\text { Serum monocyte concentration } \\
\left(\times 10^{9} / \mathrm{L}, \text { range }\right)\end{array}$ & $0.20-0.27$ & $0.27-0.32$ & $0.32-0.38$ & $0.38-0.48$ & $\geq 0.48$ & \\
\hline No. of subjects & 2390 & 995 & 649 & 912 & 896 & - \\
\hline No. of BBD & 532 & 203 & 129 & 181 & 176 & - \\
\hline Model $1^{c}$ & 1.00 (reference) & $\begin{array}{c}0.90 \\
(0.75,1.07)\end{array}$ & $\begin{array}{c}0.87 \\
(0.70,1.07)\end{array}$ & $\begin{array}{c}0.87 \\
(0.71,1.04)\end{array}$ & $\begin{array}{c}0.85 \\
(0.70,1.03)\end{array}$ & 0.06 \\
\hline Model $2^{\mathrm{d}}$ & 1.00 (reference) & $\begin{array}{c}0.87 \\
(0.72,1.04)\end{array}$ & $\begin{array}{c}0.85 \\
(0.68,1.05)\end{array}$ & $\begin{array}{c}0.85 \\
(0.71,1.03)\end{array}$ & $\begin{array}{c}0.86 \\
(0.71,1.04)\end{array}$ & 0.06 \\
\hline Model $3^{\mathrm{e}}$ & 1.00 (reference) & $\begin{array}{c}0.84 \\
(0.70,1.01)\end{array}$ & $\begin{array}{c}0.84 \\
(0.67,1.04) \\
\end{array}$ & $\begin{array}{c}0.84 \\
(0.69,1.02) \\
\end{array}$ & $\begin{array}{c}0.84 \\
(0.69,1.02) \\
\end{array}$ & 0.05 \\
\hline $\begin{array}{l}\text { Serum lymphocyte concentration } \\
\left(\times 10^{9} / \mathrm{L} \text {, range }\right)\end{array}$ & $1.30-1.60$ & $1.60-1.81$ & $1.81-2.10$ & $2.10-2.60$ & $\geq 2.60$ & \\
\hline No. of subjects & 1185 & 1206 & 1114 & 1151 & 1186 & - \\
\hline No. of BBD & 275 & 280 & 225 & 229 & 212 & - \\
\hline Model $1^{c}$ & 1.00 (reference) & $\begin{array}{c}1.00 \\
(0.83,1.21)\end{array}$ & $\begin{array}{c}0.84 \\
(0.69,1.02)\end{array}$ & $\begin{array}{c}0.82 \\
(0.67,1.00)\end{array}$ & $\begin{array}{c}0.72 \\
(0.59,0.88)\end{array}$ & $<0.001$ \\
\hline Model $2^{\mathrm{e}}$ & 1.00 (reference) & $\begin{array}{c}1.01 \\
(0.83,1.22)\end{array}$ & $\begin{array}{c}0.85 \\
(0.70,1.04)\end{array}$ & $\begin{array}{c}0.83 \\
(0.68,1.01)\end{array}$ & $\begin{array}{c}0.75 \\
(0.61,0.92)\end{array}$ & 0.001 \\
\hline Model $3^{\mathrm{f}}$ & 1.00 (reference) & $\begin{array}{c}0.99 \\
(0.82,1.2)\end{array}$ & $\begin{array}{c}0.85 \\
(0.69,1.04) \\
\end{array}$ & $\begin{array}{c}0.84 \\
(0.68,1.02) \\
\end{array}$ & $\begin{array}{c}0.75 \\
(0.61,0.92) \\
\end{array}$ & 0.002 \\
\hline Serum neutrophil-to-lymphocyte ratio (range) & $1.05-1.38$ & $1.38-1.66$ & $1.66-2.01$ & $2.01-2.68$ & $\geq 2.68$ & \\
\hline No. of subjects & 1173 & 1164 & 1168 & 1169 & 1169 & - \\
\hline No. of BBD & 241 & 228 & 228 & 264 & 260 & - \\
\hline Model $1^{\mathrm{c}}$ & 1.00 (reference) & $\begin{array}{c}0.94 \\
(0.77,1.15)\end{array}$ & $\begin{array}{c}0.94 \\
(0.77,1.15)\end{array}$ & $\begin{array}{c}1.13 \\
(0.93,1.38)\end{array}$ & $\begin{array}{c}1.11 \\
(0.91,1.35)\end{array}$ & 0.09 \\
\hline Model $2^{\mathrm{e}}$ & 1.00 (reference) & $\begin{array}{c}0.95 \\
(0.77,1.16)\end{array}$ & $\begin{array}{c}0.96 \\
(0.78,1.17)\end{array}$ & $\begin{array}{c}1.15 \\
(0.94,1.40)\end{array}$ & $\begin{array}{c}1.12 \\
(0.92,1.36)\end{array}$ & 0.08 \\
\hline Model $3^{\mathrm{f}}$ & 1.00 (reference) & $\begin{array}{c}0.93 \\
(0.76,1.14)\end{array}$ & $\begin{array}{c}0.94 \\
(0.77,1.15)\end{array}$ & $\begin{array}{c}1.11 \\
(0.91,1.36)\end{array}$ & $\begin{array}{c}1.08 \\
(0.89,1.32)\end{array}$ & 0.15 \\
\hline
\end{tabular}

${ }^{\mathrm{a}} \mathrm{BBD}$, benign breast disease. ${ }^{\mathrm{b}}$ Multiple logistic regression analysis. ${ }^{\mathrm{c}}$ Model 1. Crude. ${ }^{\mathrm{d}}$ adjusted odds ratios (95\% confidence interval) (all such values). ${ }^{\mathrm{e}} \mathrm{Model}$ 2, Age- and BMI-adjusted. ${ }^{\mathrm{f}}$ Model 3, adjusted for age, body mass index, smoking status, drinking status, fasting blood glucose, systolic blood pressure, total cholesterol, triglycerides, high-density lipoprotein, low-density lipoprotein, surgery history, menopausal status, and family history of cardiovascular disease. ${ }^{\mathrm{g}}$ Boldface indicates statistical significance $(P<0.05)$ (or appropriate value).

shown in Table 1 . Women with BBD were older than those without BBD. Subjects with BBD had lower BMI, WC, and SBP levels, were more likely to be everyday drinkers, and were less likely to be non-drinkers, and a higher proportion have had surgery $(P<0.05)$. No significant differences were observed between participants among TC, TG, LDL, HDL, DBP, FBG, smoking status, and history of CVD. 
The crude and adjusted associations between leukocyte subtypes and BBD are presented in Table 2. After final multiple adjustment, the OR (95\% CI) of BBD for leukocyte and its subtypes across the quintiles were as follows: leukocyte counts, $1.00,1.01(0.83,1.22), 0.90$ (0.73, 1.11), 0.94 $(0.77,1.16)$, and $0.92(0.75,1.14)(P$ for trend $=0.37)$; neutrophil counts, $1.00,0.89(0.73,1.09), 0.88(0.72,1.08)$, $0.91(0.74,1.11)$, and $1.01(0.82,1.24)(P$ for trend $=0.71)$; monocyte counts, $1.00,0.84(0.70,1.01), 0.84(0.67,1.04)$, $0.84(0.69,1.02)$, and $0.84(0.69,1.02)(P$ for trend $=0.05)$; lymphocyte counts, $1.00,0.99(0.82,1.2), 0.85(0.69,1.04)$, $0.84(0.68,1.02)$, and $0.75(0.61,0.92)(P$ for trend $=0.002)$; neutrophil-to-lymphocyte ratio, 1.00, $0.93(0.76,1.14), 0.94$ $(0.77,1.15), 1.11(0.91,1.36)$, and $1.08(0.89,1.32)$ ( $P$ for trend $=0.15)$, respectively. The test for interactions between menopausal status and BBD was not found to be significant after adjustment, and there were not significant interactions between the quintiles of leukocyte and its subtype counts and confounders of BBD in the final models (all $P$ values for interaction $>0.10$ ).

\section{Discussion}

This study was designed to assess the association between leukocyte and its subtypes and BBD in a general women population. The results suggested that higher lymphocyte counts are independently associated with the lower prevalence of BBD. To our knowledge, this is the first large-scale study concerning the topic of lymphocyte counts and prevalence of BBD.

To date, only a nested case-control study has investigated the association between several inflammatory markers and $\mathrm{BBD}$, and its results suggested that serum $\mathrm{C}$-reactive protein (CRP) levels were independently associated with $\mathrm{BBD}$, and adiponectin levels had an inverse association with BBD in the general women population [2]. Although leukocyte and its subtypes are simple and inexpensive markers of inflammation and associated with many diseases [20-22], no previous studies have assessed the association between leukocyte and its subtypes and BBD. The current study demonstrated that higher lymphocyte counts are independently associated with lower prevalence of BBD, whereas leukocyte and other subtypes did not demonstrate any association with BBD. More studies are needed to confirm whether these results can be observed in other populations.

Lymphocytes, especially tumor-infiltrating lymphocytes, play an important role in tumor-derived inflammatory responses [23-25]. Lymphocytes exert their antitumor activity by inducing cytotoxic cell death and inhibiting tumor proliferation [26]. Several studies have shown that increased infiltration of lymphocytes in tumor tissue predicted better survival outcomes in cancer patients $[27,28]$. Therefore, low lymphocyte counts might result in an insufficient immunological reaction, which leads to inferior survival in multiple cancers $[29,30]$. These mechanisms may partially explain our results. Further studies should be conducted to mechanistically describe the association between leukocyte and its subtypes and BBD.
The reliability and validity of these findings are strengthened by the population-based design, the large sample size, and the fact that we performed a detailed evaluation of an extensive set of potential confounders including sociodemographic, lifestyle, and clinically relevant variables. Previous studies have shown that surgery may affect the immunological and inflammatory response [31-33], resulting in a difference in leukocyte counts. Besides, the menopausal status can be an effect modifier or confounding factor for the association between breast tumor and WBC subtypes [34]. In order to avoid the influence of these variables, we adjusted them as confounding factors. Several limitations of the present study should be noted. First, this is a cross-sectional study, so we could not infer the causality between leukocyte subtypes and BBD. Therefore, further cohort studies and intervention trials should be conducted to confirm the association between leukocyte subtypes and BBD. Second, although numerous confounding factors were adjusted for during the analysis, the study cannot eliminate the potential effects of other unmeasured factors completely. Third, the use of oral contraceptives has been reported to influence the development of benign breast disease [35]. However, the present study did not collect the information on the use of oral contraceptives. Thus, the effect of oral contraceptives on the association between lymphocyte counts and benign breast disease cannot be ruled out. Finally, since we did not have specific pathological types of $\mathrm{BBD}$, we are unable to assess the association between leukocyte subtypes and specific types of BBD. A more detailed study on different subtypes of BBD is needed to better understand the association observed between leukocyte subtypes and BBD.

In conclusion, the present study firstly showed that a higher lymphocyte count is associated with a lower prevalence of $\mathrm{BBD}$. This finding suggests that lymphocyte counts might be a novel predictive factor or treatment target for developing BBD. Further prospective or clinical studies are necessary to confirm these preliminary findings.

\section{Abbreviations}

BC: $\quad$ Breast cancer

BBD: Benign breast disease

TCLSIH: Tianjin chronic low-grade systemic inflammation and health

BMI: $\quad$ Body mass index

CV: $\quad$ Coefficients of variation

WC: Waist circumference

WGOC: Working Group on Obesity in China

TC: $\quad$ Total cholesterol

TG: $\quad$ Triglycerides

LDL: Low-density lipoprotein

HDL: High-density lipoprotein

FBG: $\quad$ Fasting blood glucose

BP: Blood pressure

CI: Confidence interval

OR: Odds ratio

CVD: Cerebrovascular disease

CRP: C-reactive protein. 


\section{Data Availability}

The raw/processed data required to reproduce these findings cannot be shared at this time as the data also forms part of an ongoing study.

\section{Ethical Approval}

All procedures performed in studies involving human participants were in accordance with the ethical standards of the institutional and/or national research committee and with the 1964 Helsinki declaration and its later amendments or comparable ethical standards. This article does not contain any studies with animals performed by any of the authors.

\section{Consent}

Informed consent was obtained from all individual participants included in the study.

\section{Conflicts of Interest}

No potential conflicts of interest were disclosed.

\section{Authors' Contributions}

Y. S. and X. W. analyzed and interpreted data and wrote the manuscript. L. H., Y. G., X. C., J. F., H. W., X. L., F. K., B. S., R. S., Q. Z., L. L., G. M., S. Z., and Y. J. researched data, contributed to the discussion, and edited the manuscript. $\mathrm{K}$. $\mathrm{N}$. designed the study, contributed to the discussion, and reviewed and edited the manuscript. K. N. is the guarantor of this work and, as such, has full access to all the data in the study and takes responsibility for the integrity of the data and the accuracy of the data analysis.

\section{Acknowledgments}

The authors thank Health Management Centre of Tianjin Medical University General Hospital for their strong support and help in the field investigation. The authors appreciate the cooperation and participations of teacher, nurses, students, and participants. This study was supported by grants from the National Natural Science Foundation of China (Nos. 81673166, 81372118, 81372467, and 81302422); Tianjin Science \& Technology Plan Projects (No. 17ZXMFSY00190); Tianjin Traditional Chinese Medicine Research Project, Tianjin Health and Family Planning Commission (No. 2017003); Tianjin health and family planning high level talent selection and training project.

\section{References}

[1] M. Orditura, G. Galizia, A. Diana et al., "Neutrophil to lymphocyte ratio (NLR) for prediction of distant metastasis-free survival (DMFS) in early breast cancer: a propensity score-matched analysis," ESMO Open, vol. 1, no. 2, Article ID e000038, 2016.
[2] C. Catsburg, M. J. Gunter, C. Chen et al., "Insulin, estrogen, inflammatory markers, and risk of benign proliferative breast disease," Cancer Research, vol. 74, no. 12, pp. 3248-3258, 2014.

[3] G. Ramsey-Stewart, "The treatment of symptomatic benign breast disease with danazol," The Australian and New Zealand Journal of Obstetrics and Gynaecology, vol. 28, no. 4, pp. 299-304, 1988.

[4] E. Elinav, R. Nowarski, C. A. Thaiss, B. Hu, C. Jin, and R. A. Flavell, "Inflammation-induced cancer: crosstalk between tumours, immune cells and microorganisms," Nature Reviews Cancer, vol. 13, no. 11, pp. 759-771, 2013.

[5] R. Singh, M. K. Mishra, and H. Aggarwal, "Inflammation, immunity, and cancer," Mediators of Inflammation, vol. 2017, Article ID 6027305, 1 pages, 2017.

[6] D. S. O'Callaghan, D. O'Donnell, F. O'Connell, and K. J. O'Byrne, "The role of inflammation in the pathogenesis of non-small cell lung cancer," Journal of Thoracic Oncology, vol. 5, no. 12, pp. 2024-2036, 2010.

[7] G. Galizia, M. Orditura, C. Romano et al., "Prognostic significance of circulating IL-10 and IL-6 serum levels in colon cancer patients undergoing surgery," Clinical Immunology, vol. 102, no. 2, pp. 169-178, 2002.

[8] Z. Mei, Y. Liu, C. Liu et al., "Tumour-infiltrating inflammation and prognosis in colorectal cancer: systematic review and meta-analysis," British Journal of Cancer, vol. 110, no. 6, pp. 1595-1605, 2014.

[9] M. R. Hussein and H. I. Hassan, "Analysis of the mononuclear inflammatory cell infiltrate in the normal breast, benign proliferative breast disease, in situ and infiltrating ductal breast carcinomas: preliminary observations," Journal of Clinical Pathology, vol. 59, no. 9, pp. 972-977, 2006.

[10] C. Bickel, H. J. Rupprecht, S. Blankenberg et al., "Relation of markers of inflammation (C-reactive protein, fibrinogen, von Willebrand factor, and leukocyte count) and statin therapy to long-term mortality in patients with angiographically proven coronary artery disease," The American Journal of Cardiology, vol. 89, no. 8, pp. 901-908, 2002.

[11] H. Ekici, E. Malatyalioglu, A. Kokcu, E. Kurtoglu, M. Tosun, and H. Celik, "Do leukocyte and platelet counts have benefit for preoperative evaluation of endometrial cancer?" Asian Pacific Journal of Cancer Prevention, vol. 16, no. 13, pp. 5305-5310, 2015.

[12] C. Tibaldi, E. Vasile, I. Bernardini, C. Orlandini, M. Andreuccetti, and A. Falcone, "Baseline elevated leukocyte count in peripheral blood is associated with poor survival in patients with advanced non-small cell lung cancer: a prognostic model," Journal of Cancer Research and Clinical Oncology, vol. 134, no. 10, pp. 1143-1149, 2008.

[13] K. L. Margolis, R. J. Rodabough, and C. A. Thomson, "Prospective study of leukocyte count as a predictor of incident breast, colorectal, endometrial, and lung cancer and mortality in postmenopausal women," Archives of Internal Medicine, vol. 167, no. 17, pp. 1837-1844, 2007.

[14] Y. Gu, H. Li, X. Bao et al., "The relationship between thyroid function and the prevalence of type 2 diabetes mellitus in euthyroid subjects," The Journal of Clinical Endocrinology \& Metabolism, vol. 102, no. 2, pp. 2016-2965, 2017.

[15] R. Ohlinger, G. M. Klein, and G. Köhler, "Ultrasound of the breast-value of sonographic criteria for the differential diagnosis of solid lesions," Ultraschall in der Medizin-European Journal of Ultrasound, vol. 25, no. 1, pp. 48-53, 2004.

[16] Q.-L. Zhao, X.-N. Xia, Y. Zhang et al., "Elastosonography and two-dimensional ultrasonography in diagnosis of axillary 
lymph node metastasis in breast cancer," Clinical Radiology, vol. 73 , no. 3, pp. 312-318, 2018.

[17] H. R. Harris, S. S. Tworoger, S. E. Hankinson, B. A. Rosner, and K. B. Michels, "Plasma leptin levels and risk of breast cancer in premenopausal women," Cancer Prevention Research, vol. 4, no. 9, pp. 1449-1456, 2011.

[18] X. Hu, S. C. Juneja, and N. J. Maihle, "Leptin-a growth factor in normal and malignant breast cells and for normal mammary gland development," CancerSpectrum Knowledge Environment, vol. 94, no. 22, pp. 1704-1711, 2002.

[19] B. F. Zhou, "Predictive values of body mass index and waist circumference for risk factors of certain related diseases in Chinese adults-study on optimal cut-off points of body mass index and waist circumference in Chinese adults," Biomedical and Environmental Sciences: BES, vol. 15, no. 1, pp. 83-96, 2002.

[20] P. P. Chmielewski and B. Strzelec, "Elevated leukocyte count as a harbinger of systemic inflammation, disease progression, and poor prognosis: a review," Folia Morphologica, vol. 77, no. 2, pp. 171-178, 2018.

[21] A. Diaz-Redondo, J. Miranda-Bautista, J. García-Lledó, J. Perez Gisbert, and L. Menchén, "The potential usefulness of human leukocyte antigen typing for celiac disease screening: a systematic review and meta-analysis," Revista Española de Enfermedades Digestivas, vol. 107, no. 7, pp. 423-429, 2015.

[22] C. Golias, E. Tsoutsi, and A. Matziridis, "Review. Leukocyte and endothelial cell adhesion molecules in inflammation focusing on inflammatory heart disease," In Vivo (Athens, Greece), vol. 21, no. 5, pp. 757-769, 2007.

[23] M. J. Smyth, G. P. Dunn, and R. D. Schreiber, "Cancer immunosurveillance and immunoediting: the roles of immunity in suppressing tumor development and shaping tumor immunogenicity," Advances in Immunology, vol. 90, pp. 1-50, 2006.

[24] G. P. Dunn, L. J. Old, and R. D. Schreiber, "The immunobiology of cancer immunosurveillance and immunoediting," Immunity, vol. 21, no. 2, pp. 137-148, 2004.

[25] Y.-g. Man, A. Stojadinovic, J. Mason et al., “Tumor-infiltrating immune cells promoting tumor invasion and metastasis: existing theories," Journal of Cancer, vol. 4, no. 1, pp. 84-95, 2013.

[26] A. Mantovani, P. Allavena, A. Sica, and F. Balkwill, "Cancerrelated inflammation,” Nature, vol. 454, no. 7203, pp. 436444, 2008.

[27] N. Nguyen, E. Bellile, D. Thomas et al., "Tumor infiltrating lymphocytes and survival in patients with head and neck squamous cell carcinoma," Head \& Neck, vol. 38, no. 7, pp. 1074-1084, 2016.

[28] Y. Mao, Q. Qu, X. Chen, O. Huang, J. Wu, and K. Shen, "The prognostic value of tumor-infiltrating lymphocytes in breast cancer: a systematic review and meta-analysis," PLoS One, vol. 11, no. 4, Article ID e0152500, 2016.

[29] T. K. Hoffmann, G. Dworacki, and T. Tsukihiro, "Spontaneous apoptosis of circulating T lymphocytes in patients with head and neck cancer and its clinical importance," Clinical Cancer Research: an Official Journal of the American Association for Cancer Research, vol. 8, no. 8, pp. 2553-2562, 2002.

[30] J. P. Väyrynen, A. Tuomisto, K. Klintrup, J. Mäkelä, T. J. Karttunen, and M. J. Mäkinen, "Detailed analysis of inflammatory cell infiltration in colorectal cancer," British Journal of Cancer, vol. 109, no. 7, pp. 1839-1847, 2013.

[31] J.-X. Lin, Z.-K. Wang, Y.-Q. Huang et al., "Dynamic changes in pre- and postoperative levels of inflammatory markers and their effects on the prognosis of patients with gastric cancer," Journal of Gastrointestinal Surgery, 2020.

[32] G. Vanni, M. Materazzo, T. Perretta et al., "Impact of awake breast cancer surgery on postoperative lymphocyte responses," In Vivo, vol. 33, no. 6, pp. 1879-1884, 2019.

[33] T. Mineo, F. Sellitri, G. Vanni, F. Gallina, and V. Ambrogi, "Immunological and inflammatory impact of non-intubated lung metastasectomy," International Journal of Molecular Sciences, vol. 18, no. 7, p. 1466, 2017.

[34] B. Park, H. S. Lee, J. W. Lee, and S. Park, "Association of white blood cell count with breast cancer burden varies according to menopausal status, body mass index, and hormone receptor status: a case-control study," Scientific Reports, vol. 9, no. 1, p. 5762, 2019.

[35] M. Vessey and D. Yeates, "Oral contraceptives and benign breast disease: an update of findings in a large cohort study," Contraception, vol. 76, no. 6, pp. 418-424, 2007. 